\title{
ON A TYPE OF PLANE UNICURSAL CURVE
} BY HAROLD HILTON

The type considered is that which meets the sides of a real triangle in points whose parameters have at most three distinct values. If we take the triangle as triangle of reference, and suitable homogeneous coordinates, we may suppose any point $(x, y, z)$ on the curve given by the equations

where

$$
\left\{\begin{array}{l}
x=(t-\alpha)^{p_{1}}(t-\beta)^{q_{1}}(t-\gamma)^{r_{1}}, \\
y=(t-\alpha)^{p_{2}}(t-\beta)^{q_{2}}(t-\gamma)^{r_{2}}, \\
z=(t-\alpha)^{p_{3}}(t-\beta)^{q_{3}}(t-\gamma)^{r_{3}},
\end{array}\right.
$$

$$
p_{1}+q_{1}+r_{1}=p_{2}+q_{2}+r_{2}=p_{3}+q_{3}+r_{3}=n,
$$

where $n$ is the degree of the curve.

In this, one of the quantities $p_{1}, p_{2}, p_{3}$ is zero and the other two are zero or positive integers; and so for $q_{1}, q_{2}, q_{3}$ and $r_{1}, r_{2}, r_{3}$.

We may denote the curve (1) symbolically by the array

$$
\left|\begin{array}{lll}
p_{1} & p_{2} & p_{3} \\
q_{1} & q_{2} & q_{3} \\
r_{1} & r_{2} & r_{3}
\end{array}\right|
$$

or, as it may be written to save space,

$$
\left(\begin{array}{lllllllll}
p_{1} & q_{1} & r_{1}, & p_{2} & q_{2} & r_{2}, & p_{3} & q_{3} & r_{3}
\end{array}\right) .
$$

It is evident that $(a)$ each row of (3) contains at least one zero, $(b)$ the sum of the elements in each column is $n$, (c) no two columns are identical, (d) we get no essentially distinct curve, if we interchange two rows or interchange two columns.

The first problem that suggests itself is to find the number of essentially distinct curves of the required type; $i$. e., to find the number of distinct arrays (3) subject to the restrictions just mentioned. We content ourselves with giving the 
result in the form of a table, the first column giving the array (4), and the last two giving the number of distinct curves when $n$ is odd or even.

Note that in this table

$$
\left\{\begin{array}{r}
p+P=q+Q=n, \\
\lambda+\mu+\nu=n, \\
f+F=g+G=h+H=n ;
\end{array}\right.
$$

$f$ is not less than $F, g, G, h$, or $H$.

\begin{tabular}{|c|c|c|c|}
\hline Array & & $n$ odd & $n$ even \\
\hline$(n 00,0 n 0,00 n)$ & & 1 & 1 \\
\hline$(p P 0,0 n 0,00 n)$ & & $n-1$ & $n-1$ \\
\hline$(0 p P, 0 n 0,00 n)$ & $p \geqq P$ & $\frac{1}{2}(n-1)$ & $\frac{1}{2} n$ \\
\hline$(\lambda \mu \nu, 0 n 0,00 n)$ & $\mu \geqq \nu$ & $\frac{1}{4}(n-1)^{2}$ & $\frac{1}{4} n(n-2)$ \\
\hline$(p P 0, q Q 0,00 n)$ & $p \geqq Q ; p>P, q$ & $\frac{1}{4}(n-1)^{2}$ & $\frac{1}{4} n(n-2)$ \\
\hline$(0 p P, q Q 0,00 n)$ & & $(n-1)^{2}$ & $(n-1)^{2}$ \\
\hline$(\lambda \mu \nu, q Q 0,00 n)$ & $\begin{array}{l}\lambda>\mu \quad \text { or } \\
\lambda=\mu, q>Q\end{array}$ & $\frac{1}{4}(n-1)^{2}(n-2)$ & $\frac{1}{4} n(n-2)^{2}$ \\
\hline$(f F 0, f 0 F, 0 F f)$ & $f>F$ & $\frac{1}{2}(n-1)$ & $\frac{1}{2}(n-2)$ \\
\hline$(f F 0, F 0 f, 0 f F)$ & $f>F$ & $\frac{1}{2}(n-1)$ & $\frac{1}{2}(n-2)$ \\
\hline$(f f 0, f 0 f, 0 f f)$ & & 0 & \\
\hline$(f F 0, f 0 F, 0 h H)$ & $f>F ; f>h \geqq H$ & $\frac{1}{8}(n-1)(n-3)$ & $\frac{1}{8} n(n-2)$ \\
\hline$(f F 0, F 0 f, 0 h H)$ & & $\frac{1}{4}(n-1)(n-3)$ & $\frac{1}{4}(n-2)^{2}$ \\
\hline$(f F 0, g 0 G, 0 F f)$ & $f>F ; f>g \geqq G$ & $\frac{3}{8}(n-1)(n-3)$ & $\frac{1}{8} n(n-2)$ \\
\hline$(f F 0, g 0 G, 0 g G)$ & $f>g, G ; g \neq G$ & $\frac{1}{4}(n-1)(n-3)$ & $\frac{1}{4}(n-2)(n-4)$ \\
\hline$(f F 0, g 0 G, 0 G g)$ & $f>g, G ; g \neq G$ & $\frac{1}{4}(n-1)(n-3)$ & $\frac{1}{4}(n-2)(n-4)$ \\
\hline$(f F 0, g 0 g, g 0 g)$ & & & $\frac{1}{2}(n-2)$ \\
\hline$(f F 0, g 0 G, 0 h H)$ & & $\frac{1}{6}(n-1)(n-3)(n-5)$ & $\frac{1}{6}(n-2)(n-3)(n-4)$ \\
\hline
\end{tabular}

There are in all $\frac{1}{12} n\left(5 n^{2}+7\right)$ distinct curves, if $n$ is odd, and $\frac{5}{12} n\left(n^{2}+2\right)$, if $n$ is even. In this, however, may be included some curves of degree less than $n$ if two rows of (3) are proportional. This can only be the case, if the curve belongs to the type $(0 p P, 0 n 0,00 n)$ and $p$ and $n$ have a common factor, or to the type $(\lambda \mu \nu, q Q 0,00 n)$ and $q$ is a multiple of $n^{\prime}$, where $\nu / n=\nu^{\prime} / n^{\prime}$ and $\nu^{\prime}$ is prime to $n^{\prime}$.

For $n=3$, there are 13 cubics, 4 cuspidal, 4 crunodal, and 5 acnodal. 
For $n=4$, there are 29 quartics, excluding $(022,040,004)$, which is a conic. Of these, 8 have a superlinear branch of order three, 3 have a cusp-linear triple point, 6 have a rhamphoid cusp and another double point, 2 have a crunode and two acnodes, 2 have a cusp and two acnodes, 2 have two cusps and an acnode, 1 has three cusps, and 5 have three acnodes.

Among them we may note the following, whose equations are given referred to the triangle with its vertices at the double points:

(211, 040, 004), $3\left(y^{2} z^{2}+z^{2} x^{2}+x^{2} y^{2}\right)+2 x y z(2 \sqrt{2} x$

$(310,130,004)$, $+2 \sqrt{2} y+z)=0$ (x) $(031,220,004), \quad z^{2}(x-y)^{2}-4 x y z(x+y)+16 x^{2} y^{2}=0$, $(211,130,004), z\{3(x+y)+\sqrt{5}(x-y)\}\{(x+y) z+x y\}$ $+2 x^{2} y^{2}=0$ $(310,202,013), \quad y^{2} z^{2}+z^{2} x^{2}+x^{2} y^{2}+x y z(x-2 y-2 z)=0$, $(310,202,022), \quad y^{2} z^{2}+z^{2} x^{2}+x^{2} y^{2}-2 x y z(x+y)=0$.

It will be noticed that the curve obtained by the quadratic transformation, which consists in replacing $x, y, z$ by $1 / x$, $1 / y, 1 / z$ in (1), is a curve of the same type.

Without loss of generality we may suppose that in (1) the numerically greatest of $p_{2}-p_{3}, p_{3}-p_{1}, \ldots, r_{1}-r_{2}$ is $r_{3}-r_{1}$, and that $r_{3} \geqq r_{2} \geqq r_{1}=0, p_{1}-p_{3} \geqq q_{1}-q_{3}$. We may then put (1) in the form

$$
x=t^{a}(t-1)^{b}, y=t^{c}(t-1)^{d}, z=1,
$$

on replacing $t$ by

$$
\{\gamma(\beta-\alpha) t-\alpha(\beta-\gamma)\} \div\{(\beta-\alpha) t-(\beta-\gamma)\},
$$

and replacing $x, y, z$ by suitable multiples of themselves.

In (6) we have

(7) $a=p_{1}-p_{3}, b=q_{1}-q_{3}, c=p_{2}-p_{3}, d=q_{2}-q_{3}$,

(8) $a \geqq b \geqq 0,(a+b) \geqq(c+d) \geqq 0, b+c \geqq 0, a+d \geqq 0$,

in virtue of the restrictions which we have supposed to hold between $p_{1}, p_{2}, \ldots, r_{3}$. 
The sides of the triangle of reference meet the curve only where $t=0,1$, or $\infty$. We shall suppose excluded the case $a d=b c$, when the curve becomes the well known type

The tangent to (6) is

$$
x: y: z=t^{a}: t^{c}: 1 \text {. }
$$

(9) $t^{-a}(t-1)^{-b}\{(c+d) t-c\} x-t^{-c}(t-1)^{-a}\{(a+b) t-a\} y$

$$
+\{(a+b-c-d) t-(a-c)\} z=0 .
$$

The inflexions, other than $t=0,1, \infty$, are given by

$$
\begin{array}{r}
\{(a+b-c-d) t-(a-c)\}\{(a+b) t-a\}\{(c+d) t-c)\} \\
+(a d-b c) t(t-1)=0 .
\end{array}
$$

We see that the degree $n$ of (6) is $a+b$ if $c \geqq 0, d \geqq 0$; $a+b-d$ if $c \geqq 0, d \leqq 0 ; a+b-c$ if $d \geqq 0, c \leqq 0$.

The class is in general the greatest of

$$
a+b+1, a+d+1, b+c+1 \text {. }
$$

It may be less than this by unity in certain cases, e. g., if $a+b=c+d$.

The knowledge of the degree and class, combined with the fact that the deficiency is zero, gives the Plücker's numbers of the curve.

If $A B C$ is the triangle whose sides are met by (6) in points whose parameters are $0,1, \infty$, we have from (9) and (10) the following result.

From each of $A, B, C$ at most one tangent can be drawn touching the curve at a point not lying on a side of the triangle $A B C$, and the number of these tangents is equal to the number of inflexions which do not lie on the sides of the triangle.

This number is three at most, but can be two, one, or zero, if one or more of

$$
a+b-c-d, c+d, b, c, d, a-c, b-d
$$

is zero. For instance, the type $(f f 0, f 0 f, 0 f f)$, in which $c+d, b$, and $a-c$ are zero, has no inflexion except at the vertices of $A B C$. It is a curve with three superlinear branches of order $f$, and includes the case ot the threecusped hypocycloid. 
The only other type with no inflexion except those on a side of the triangle $A B C$ is $(n 00,0 n 0,00 n)$, discussed in Messenger of Mathematics, vol. 49 (1920), p. 132. Another interesting type is $(f F 0, F 0 f, 0 f F)$, discussed in this Bulletis, vol. 29 (1923), p. 303 . We may also note the type $(f F 0, F f 0,00 n)$ with two real inflexions if $f \geqq \frac{1}{2}(n+\sqrt{n})$, especially the case in which $f=n-1$.

The number of sextactic points not on a side of the triangle $\mathrm{ABC}$ is twelve or less.

If a node of (6) has parameters $t_{1}$ and $t_{2}($ not 0,1, or $\infty$ ) and

$$
t_{1} / t_{2}=\omega, \quad\left(t_{1}-1\right) /\left(t_{2}-1\right)=\Omega,
$$

we have $\omega$ and $\Omega \neq 1, \omega \neq \Omega$, while

$$
\omega^{a} \Omega^{b}=1, \omega^{c} \Omega^{d}=1 \text {. }
$$

These give

$$
\omega^{\varepsilon}=1, \Omega^{\varepsilon}=1, \text { where } \varepsilon=a d-b c,
$$

whence

$$
t_{1}=\omega(1-\Omega) /(\omega-\Omega), t_{2}=(1-\Omega) /(\omega-\Omega) .
$$

It is at once seen that the values of $t_{1}$ and $t_{2}$ given by (12), (13), (14) are complex and that the corresponding values of $x$ and $y$ given by (6) are real. Hence we have the following result.

The only point-singularities of the curve other than those on the sides of the triangle $A B C$ are real acnodes.

If we take

$$
\boldsymbol{\omega}=e^{2 h \pi i / \varepsilon}, \boldsymbol{\Omega}=e^{2 k \pi i / \varepsilon},
$$

the equation (12) becomes

$$
h a+k b \equiv 0(\bmod \varepsilon), h c+k d \equiv 0(\bmod \varepsilon)
$$

and the acnodes are given on finding values of $h$ and $k$ between 1 and $\varepsilon-1$ satisfying (16). We may simplify (16) by putting

$a=\lambda a_{1}, c=\lambda c_{1}, b=\mu b_{1}, d=\mu d_{1}, \varepsilon=\lambda \mu \varepsilon_{1}=\lambda \mu\left(a_{1} d_{1}-b_{1} c_{1}\right)$, where $a_{1}$ is prime to $c_{1}$ and $b_{1}$ to $d_{1}$. We readily find 
from (16), on eliminating $h$ or $k$, that $h=\mu h_{1}, k=\lambda k_{1}$, where $h_{1}$ and $k_{1}$ are integral, and we get

$$
h_{1} a_{1}+k_{1} b_{1} \equiv 0\left(\bmod \varepsilon_{1}\right), h_{1} c_{1}+k_{1} d_{1} \equiv 0\left(\bmod \varepsilon_{1}\right) \text {. }
$$

The nature of the singularities on the sides of the triangle $A B C$ is readily determined. For instance, suppose in (6) $c>a>0$. Then (6) gives an expansion for $t$ in ascending powers of $x^{1 / a}$, and thence we get for $y$ an expansion of the form

$$
y=x^{c / a}\left(\alpha+\beta x^{1 / a}+\gamma x^{2 / a}+\cdots\right)
$$

in general, fixing the nature of the singularity for which $t$ is zero.

Bedford College, University of London

\section{SURFACES WITH ORTHOGONAL LOCI OF THE CENTERS OF GEODESIC CURVATURE OF AN ORTHOGONAL SYSTEM*}

BY MALCOLM FOSTER

We consider a surface $S$ referred to any orthogonal system. Let $G_{1}$ and $G_{2}$ be the centers of geodesic curvature of the curves $u=$ const. and $v=$ const. respectively, through any point $M$ of $S$. As $M$ is displaced over the entire surface the loci of $G_{1}$ and $G_{2}$ will in general be two surfaces $S_{1}$ and $S_{2}$, corresponding elements of which are those which result from a common displacement of $M$. We ask: What are the surfaces $S$ for which the surfaces $S_{1}$ and $S_{2}$ correspond with orthogonality of linear elements?

The condition that the displacements of $G_{1}$ and $G_{2}$ be orthogonal for every displacement of $M$, is that the absolute displacements of these points in the directions of the axes of the moving trihedral at $M$ satisfy the relation

$$
\sum \delta x_{1} \delta x_{2}=0
$$

\footnotetext{
* Presented to the Society, April 28, 1923.
} 